Cahiers d'études africaines

\title{
HATZKY, Christine. - Cubans in Angola : South-South Cooperation and Transfer of Knowledge, 1976-1991
}

\section{Constantin Katsakioris}

\section{OpenEdition}

\section{Journals}

Édition électronique

URL : http://journals.openedition.org/etudesafricaines/20773

DOI : 10.4000/etudesafricaines.20773

ISSN : 1777-5353

\section{Éditeur}

Éditions de l'EHESS

\section{Édition imprimée}

Date de publication : 1 juillet 2017

Pagination : 452-465

ISBN : 978-2-7132-2688-5

ISSN : 0008-0055

\section{Référence électronique}

Constantin Katsakioris, " Hatzkr, Christine. - Cubans in Angola: South-South Cooperation and Transfer of Knowledge, 1976-1991 », Cahiers d'études africaines [En ligne], 226 | 2017, mis en ligne le 01 avril 2017, consulté le 24 septembre 2020. URL : http://journals.openedition.org/etudesafricaines/20773 DOI : https://doi.org/10.4000/etudesafricaines.20773

Ce document a été généré automatiquement le 24 septembre 2020.

(C) Cahiers d'Études africaines 


\title{
HATZKY, Christine. - Cubans in Angola : South-South Cooperation and Transfer of Knowledge, 1976-1991
}

\author{
Constantin Katsakioris
}

\section{RÉFÉRENCE}

HATZKY, Christine. - Cubans in Angola : South-South Cooperation and Transfer of Knowledge, 1976-1991. Madison, The University of Wisconsin Press, 2015, 386 p., bibl.

1 De la présence d'Ernesto Che Guevara dans les maquis lumumbistes engagés contre le régime de Mobutu dans les années 1960, au soutien militaire apporté sur le terrain par Cuba au Mouvement populaire de libération de l'Angola (MPLA), dans sa guerre contre l'UNITA (Union nationale pour l'indépendance totale de l'Angola) de Jonas Savimbi, soutenue par les États-Unis et l'Afrique du Sud, c'est l'interventionnisme militaire qui a été le plus souvent retenu dans les analyses de la politique africaine de Cuba. Celle-ci s'affichait pourtant dans plusieurs autres domaines parmi lesquels la santé et l'éducation restent aujourd'hui encore les plus marquants. C'est ainsi l'un des mérites du livre de Christine Hatzky que de nous sortir de ce déterminisme guerrier en ouvrant la voie à une autre facette de la coopération cubaine en Afrique, celle de l'éducation.

Dans Cubans in Angola, elle examine en effet la coopération civile dans le domaine de l'enseignement entre Cuba et l'Angola post-colonial, depuis 1976, date de l'arrivée des premiers coopérants dans la province de Cabinda, jusqu'en 1991, lorsque civils et militaires cubains ont quitté le pays. Initialement publié en langue allemande par les éditions Oldenbourg ${ }^{17}$ en 2012, le livre a reçu le prix "Geisteswissenschaften International » pour sa contribution dans le domaine des sciences humaines et sociales, ce qui a permis sa traduction en anglais ${ }^{18}$.

3 Historienne de l'Amérique latine, C. Hatzky se donne pour tâche d'écrire l'histoire de la coopération éducative entre les deux pays, de la replacer dans le contexte 
international, mais tout d'abord d'approfondir les prémisses et les motivations des deux alliés : d'un côté Cuba et, de l'autre, l'Angola du MPLA. Dès l'introduction, elle souligne qu'il s'agissait d'une « coopération » et non pas d'une intervention militaire et civile cubaine ou soviéto-cubaine, comme plusieurs analystes avaient tenté de le créditer à l'époque de la Guerre froide. Le MPLA constituait, souligne-t-elle, à la fois l'acteur politique et le bénéficiaire de cette coopération. Pour cela, il lui a fallu réaliser un travail colossal dans les archives des deux pays. De 2004 à 2009, l'auteure a ainsi dépouillé les documents archivés auprès du ministère de l'Enseignement de l'Angola, ainsi que certains documents des archives cubaines auxquelles elle n'a cependant eu qu'un accès très limité. Dans le même temps, elle a réalisé 127 entretiens, biographiques pour la plupart, auprès de 139 témoins, dont 106 coopérants cubains (lors de 95 entretiens) et 33 experts - fonctionnaires, responsables - ou anciens élèves angolais (lors de 32 entretiens). Elle a enfin recouru à une très vaste littérature en plusieurs langues, allant des travaux plus anciens du politiste John Marcum à ceux de Christine Messiant et de Piero Gliejeses ${ }^{19}$. Ceci lui a fourni un précieux appui pour la première partie de son étude, laquelle représente près de la moitié de l'ouvrage, qui relate l'histoire des deux pays depuis leurs révolutions respectives (1959 à Cuba et 1961 en Angola), l'état de l'enseignement, les motifs de la coopération et les stratégies cubaines pour recruter des coopérants. La deuxième partie approfondit l'examen de la coopération éducative en analysant les programmes, les motivations des acteurs, tandis que la troisième s'organise autour des mémoires des témoins, cubains et angolais.

4 Si l'on retrouve, ça et là, quelques redondances, fort justifiées au demeurant par le fait que l'auteure doit condenser dans 400 pages une histoire internationale complexe, avec de nombreux acteurs (notamment en Angola) et des allées et venues permanentes entre Cuba et l'Angola, l'ouvrage reste d'une grande lisibilité et d'une écriture limpide.

5 La première partie, peut-être un peu longue pour le spécialiste, est néanmoins indispensable pour expliquer les prémisses politiques et idéologiques, et plus généralement les raisons qui ont donné naissance à la coopération. La situation très complexe à l'intérieur de l'Angola, notamment le combat entre MPLA et UNITA, les premiers contacts entre Cuba et le MPLA, le soutien militaire décisif que Cuba a apporté au MPLA en 1975-1976, les rapports entre Fidel Castro et Agostinho Neto sont analysés par le menu. La place importante de la coopération éducative dans la stratégie nationale du MPLA et dans l'ensemble des liens entre Cuba et MPLA est également examinée. C. Hatzky souscrit aux propos d'un de ses témoins, Antonio Burity da Silva, à l'époque responsable de la sélection des Angolais partant étudier à Cuba et ensuite ministre de l'Enseignement de l'Angola, qui décrit la coopération comme un « internationalisme aux bénéfices réciproques» (p.14). De fait, elle ne minimise pas la dimension internationaliste qui était mise en avant par les dirigeants cubains et qui a motivé plusieurs des coopérants (au nombre de 50000 environ) et notamment les 8000 à 10000 enseignants et spécialistes cubains sur lesquels elle se concentre. En même temps, elle fournit des informations détaillées sur les rémunérations des coopérants cubains et sur les aspects financiers de la coopération. Leurs salaires se situaient dans la moyenne de ceux des autres coopérants étrangers. Ils étaient pourtant directement payés par l'État angolais à l'État cubain - la moitié de la somme vraisemblablement en dollars - tandis que sur place les coopérants recevaient dix dollars par mois. Comme l'État cubain versait un salaire en pesos aux familles des coopérants qui étaient restées à Cuba, on peut déduire qu'il tirait aussi profit de cette coopération. Seuls les étudiantsenseignants, qui faisaient leur "stage » en Angola pour un ou deux ans, n'étaient 
apparemment pas payés par la partie angolaise. Quant aux Angolais qui partirent étudier à Cuba, que ce soit à l'université ou dans les écoles primaires et secondaires de l'Ile de la jeunesse (Isla de la Juventud), ils ont tous étudié aux frais de l'État cubain.

6 Les deuxième et troisième parties relatent les formes et l'évolution de la coopération, ainsi que les expériences et relations personnelles à travers des témoignages. C. Hatzky souligne que les coopérants cubains qui, sur place, étaient sous les auspices de l'administration civile cubaine, fonctionnaient de manière très indépendante et le plus souvent constituaient un groupe séparé des Angolais. Elle note la mutation suivante : tandis qu'au départ les coopérants cubains avaient comme mission de réorganiser l'enseignement angolais et de former les futurs enseignants, très vite, à la demande du MPLA, ils arrivèrent en masse et devinrent les enseignants les plus nombreux. Si leur travail était en général très apprécié, la conséquence fut, selon C. Hatzky, que la coopération ne servit pas la cause de la formation des enseignants angolais, mais créa une nouvelle dépendance, cette fois-ci, par rapport à la coopération cubaine. Dominant numériquement et grâce à leurs connaissances, les Cubains marquèrent l'enseignement angolais en introduisant des méthodes interactives, en établissant les programmes d'enseignement et en formant dans des conditions extrêmement difficiles la première génération post-coloniale du pays. Outre leur contribution sur le plan éducatif, leur rôle politique et le soutien qu'ils apportèrent au MPLA furent déterminants, conclut C. Hatzky. Si les Angolais gardèrent, en règle générale, les meilleurs souvenirs de leurs enseignants cubains, ces derniers, comme l'auteure le démontre dans la troisième partie, revenaient souvent traumatisés à Cuba. Victimes de la guerre ou de l'hostilité d'une partie de la population qui les voyait comme représentants du MPLA, les enseignants cubains affrontaient souvent le danger et la violence dans un pays postcolonial où les élites corrompues et enrichies optèrent finalement pour le capitalisme. Pour les Cubains, ces expériences les ont persuadés que leur pays était finalement beaucoup plus juste socialement et donc plus avancé que l'Angola. Elles leur ont aussi laissé l'amertume de sacrifices inutiles qui auront finalement été très vite oubliés.

7 Le livre de C. Hatzky est une tentative de prise en compte exhaustive des questions soulevées par cette coopération, qu'elles soient idéologiques et politiques, économiques ou humaines. Bien évidemment, on ne saurait s'attendre à trouver des réponses complètes à toutes ces interrogations, d'autant plus que les témoins cubains ont encore souvent peur de s'exprimer librement, que l'accessibilité aux archives angolaises reste partielle et que l'ouverture des archives cubaines demeure extrêmement réduite. Certes, le lecteur aurait sans doute voulu disposer de plus d'informations sur certaines questions que C. Hatzky soulève au passage, mais qu'elle n'a pu développer, par exemple sur les rapports entre la coopération éducative et la coopération technique ou sur les contrats signés entre les entreprises cubaines et l'État angolais pour la construction des écoles ou des logements de coopérants. Par ailleurs, on aurait voulu en savoir davantage sur l'une des conclusions à laquelle $\mathrm{C}$. Hatzky aboutit et qui est révélatrice des ambigüités de l'aide et de toutes les formes de coopération. Il s'agit d'expliquer comment la présence des enseignants cubains en Angola a finalement entravé la formation des enseignants angolais et de quelle manière une nouvelle dépendance fut créée. Malheureusement, les données sur l'évolution des effectifs des enseignants angolais qui auraient justifié une telle conclusion font défaut. D'ailleurs, la remarque selon laquelle plusieurs Angolais formés par les Cubains ont réussi dans les affaires ou ont occupé des postes-clés dans différentes sphères de l'État devrait conduire à une étude plus systématique des devenirs et des carrières des Angolais 
formés à Cuba. Tout bien considéré, Cubans in Angola constitue un ouvrage de référence, solide et extrêmement riche, qui ouvre la voie à de nouvelles recherches.

\section{NOTES}

17. С. Н AтZKY, Kubaner in Angola: Süd-Süd-Kooperation und Bildungstransfer 1976-1991, Munich, Oldenbourg Verlag, 2012.

18. Le livre a été revu à l'occasion de cette traduction.

19. J. Marcum, The Angolan Revolution, v. 1 \& v. 2, Cambridge, Mass., The MIT Press, 1962-1978; C. MEsSiant, L'Angola postcolonial, v. 1 \& v. 2, Paris, Karthala, 2008; P. GLIEJESES, Conflicting Missions: Havana, Washington, and Africa, 1959-1976, Chapel Hill, University of North Carolina Press, 2002. 